\title{
Distribution and molecular characteristics of rickettsiae found in ticks across Central Mongolia
}

\author{
Bazartseren Boldbaatar ${ }^{1 \dagger}$, Rui-Ruo Jiang ${ }^{2 \dagger}$, Michael E. von Fricken ${ }^{3,4^{*}} \mathbb{D}$, Sukhbaatar Lkhagvatseren ${ }^{1}$, \\ Pagbajab Nymadawa ${ }^{5}$, Bekhochir Baigalmaa ${ }^{6}$, Ya-Wei Wang ${ }^{2}$, Benjamin D. Anderson ${ }^{3}$, Jia-Fu Jiang ${ }^{2}$ \\ and Gregory C. Gray ${ }^{3}$
}

\begin{abstract}
Background: Little is known regarding tick-borne diseases in Mongolia, despite having $26 \%$ of the population still living nomadic pastoral lifestyles. A total of 1497 adult unfed ticks: 261 Ixodes persulcatus, 795 Dermacentor nuttalli, and 441 Hyalomma asiaticum, were collected from three ecologically distinct regions in Central Mongolia. Tick pools ( $n=299$ ) containing $\sim 5$ ticks each, were tested for Rickettsia and Tick-borne encephalitis virus (TBEV) using nested polymerase chain reaction, reverse transcription-PCR, and quantitative real-time RT-PCR.

Results: Assays yielded pooled prevalence of $92.5 \%$ (49/53) and 1.9\% (1/53) for pooled I. persulcatus testing positive for "Candidatus Rickettsia tarasevichiae" and TBEV, respectively, while Rickettsia raoultii was found in 72.8\% (115/158) of pooled D. nuttalli samples. When calculating a maximum likelihood estimation, an estimated 46.6\% (95\% Cl: 35.2-63.6\%) of I. persulcatus ticks in the pooled sample were infected with "Candidatus R. tarasevichiae".

Conclusions: Both "Candidatus R. tarasevichiae" and R. raoultii are recognized as emerging tick-borne pathogens, with this being one of the first reports of "Candidatus R. tarasevichiae" in Mongolia. Given that "Candidatus R. tarasevichiae" shares the same vector (I. persulcatus) as TBEV, and infections may present with similar symptoms, Mongolian physicians treating suspected cases of TBEV should include "Candidatus R. tarasevichiae" infection in their differential diagnosis and consider prescribing antimicrobial therapy.
\end{abstract}

Keywords: Rickettsia, Mongolia, Ixodes persulcatus

\section{Background}

In recent years we have become increasingly aware of the diversity of pathogens that ticks may transmit to humans or animals [1-5]. Pathogen transmission typically occurs when humans or other animals come into contact with infected ticks, some of which feed indiscriminately on vertebrate hosts. Consequently, occupations or populations that frequently enter tick habitats are at an increased risk of tick-borne disease. This holds true in Mongolia, where pastoral-herders, who make up

\footnotetext{
* Correspondence: mvonfric@gmu.edu

${ }^{\dagger}$ Equal contributors

${ }^{3}$ Division of Infectious Disease, Duke Global Health Institute, Duke University, Durham, NC, USA

${ }^{4}$ Department of Global and Community Health, George Mason University,

Fairfax, VA, USA

Full list of author information is available at the end of the article
}

$26 \%$ of the country's population of 3 million, spend prolonged periods of each day outdoors moving livestock from pasture to pasture. Despite this, little is known about the scope of tick-borne disease in Mongolia. While epidemiological studies examining spotted fever group (SFG) Rickettsia have been conducted [6-8], few have employed molecular techniques. Several additional studies have examined tick-borne encephalitis virus (TBEV), a serious tick disease found in northern Mongolia [9-12]. As a number of rural Mongolians have developed severe illnesses subsequent to tick bites, at the request of the Ministry of Health we conducted a survey for ticks in three ecologically unique regions: forested taiga, central steppe grassland, and the Gobi region of Mongolia. By implementing molecular detection methods, we sought to describe the genetic profiles 
and distribution of SFG Rickettsia among ticks located in varying ecotones and latitudes, across central Mongolia.

\section{Methods}

A total of 1497 adult unfed ticks: 261 Ixodes persulcatus, 795 Dermacentor nuttalli, and 441 Hyalomma asiaticum, were collected from three provinces; Selenge, Tov and Dornogovi, which stretch vertically across central Mongolia. Nineteen sites were selected for sampling based on their diverse landscape, elevation (range 691$1616 \mathrm{~m}$ above sea level), vegetation coverage (Table 1), and proximity to previous sampling locations of ongoing human and livestock tick-borne pathogen surveillance. Basic information pertaining to tick habitat was inferred by using annual amplitude of vegetation coverage (Normalized Difference Vegetation Index) linked to sample GPS points, with tick species and vegetation coverage depicted in Fig. 1. [13].

Sampling occurred between April $24^{\text {th }}$ and May $16^{\text {th }}$ 2015, with 884 unfed ticks collected from the environment by dragging, and an additional 613 unfed ticks removed from livestock returning from pasture. All immature life stages of ticks and engorged or partially blood fed adult ticks were excluded from analysis, as some pathogens are killed by host complement system during tick feeding.

After collection, live ticks were stored at $-80{ }^{\circ} \mathrm{C}$ in vials separated by genus (based on visual identification of morphological characteristics) and sampling location. A total of 299 pools of five ticks each $( \pm 1)$ were aggregated by tick genus and sampling site. Ticks were then homogenized with sterile mortar and pestle, with homogenate centrifuged in PBS to collect supernatant. Genomic DNA and RNA were manually extracted from tick supernatant using TaKaRa kits (TaKaRa Bio Inc, Shiga, Japan), according to the manufacturer's instructions. Species were then identified using the partial mitochondrial $16 \mathrm{~S}$ rRNA gene [14] targeting Ixodes, Hyalomma and Dermacentor tick DNA.

A nested PCR assay was used to amplify the outer membrane protein A (ompA) and citrate synthase ( $g l t A)$ gene, using the Thermo Scientific DreamTaq Green PCR Master Mix (Thermo Scientific, Waltham, USA). Primer for ompA include $\operatorname{Rr} 190.70 \mathrm{p} / \operatorname{Rr} 190.602 \mathrm{n}$ (530 bp) outer primers and 190.70-38s1/190.602-384r1 (340 bp) inner primers, which have been used in similar studies [15-18]. Primers for gltA include CS2d/CSEndr outer primers (1300 bp) and RpCS877p/RpCS1258n (381 bp) inner primers [16]. Amplified product was visualized on a 1.2\% $(w / v)$ agarose gel using gel electrophoreses, with positive amplicons directly sequenced on a 3730 Sequencer (Applied Biosystems, Carlsbad, USA) by a commercial company (Tianyi, Beijing, China). The assembled sequences were compared against the NCBI $n t / n r$ database (PubMed website: https://blast.ncbi.nlm.nih.gov/Blast.cgi) and later submitted to GenBank. Phylogenetic analysis was conducted using Molecular Evolutionary Genetics Analysis (MEGA) software, version 5.

RNA from I. persulcatus and D. nuttalli tick pools collected from Selenge and Tov aimags, were also screened for TBEV on the 7500 Real-Time PCR System (Applied Biosystems, Waltham, USA), using a commercial real-time PCR (qRT-PCR) kit provided by Liferiver ${ }^{\text {tm }}$, (Shanghai ZJ Bio-Tech Co., LTD, Shanghai, China), in which TBEV RNA was transcribed into cDNA and then amplified, according to the manufacturer's instructions. A maximum likelihood estimate (MLE) and a minimum infection rate (MIR) were calculated using PooledinRate software (http://www.cdc.gov/westnile/resource pages/mosqSurvSoft.html) to predict pool infection rates based on number of ticks per pool and infection status of pool.

\section{Results and discussion}

Overall pool detection prevalence in I. persulcatus ticks was 92.5\% (49/53) for "Candidatus R. tarasevichiae" and $1.9 \%(1 / 53)$ for TBEV (Table 2). Rickettsia raoultii was found in $72.8 \%(115 / 158)$ of $D$. nuttalli pools, with Terelj, a scenic area with high rates of international tourism, having 60/71 (84.5\%) pools testing positive. All sequences were submitted to GenBank (accession numbers KU361212-KU361217).

The MLE and MIR of $R$. raoultii in D. nuttalli ticks and "Candidatus R. tarasevichiae" in I. persulcatus ticks can be found in Table 3. Briefly, calculations of MLE for R. raoultii in D. nuttalli ticks ranged from 15.1 to $30.4 \%$ with lower rates observed in the south compared to a

Table 1 Summary of unfed adult ticks and their environment's characteristics including elevation above sea level and Normalized Difference Vegetation Index (NDVI), collected in Mongolia, from April 24 th to May $16^{\text {th }} 2015$

\begin{tabular}{lllll}
\hline Aimag & Tick species & Ticks/pools & Mean NDVI annual amplitude (range) & Elevation above sea level (m) \\
\hline Tov & Dermacentor nuttalli & $358 / 71$ & $208.5(205-249)$ & $1500-1615$ \\
Selenge & D. nuttalli & $169 / 34$ & $266.6(242-351)$ & $691-931$ \\
& Ixodes persulcatus & $261 / 53$ & $261(255-351)$ & $809-931$ \\
Dornogovi & D. nuttalli & $268 / 54$ & $89.1(58-112)$ & $895-1331$ \\
& Hyalomma asiaticum & $441 / 88$ & $21(20-61)$ & $757-929$ \\
\hline
\end{tabular}




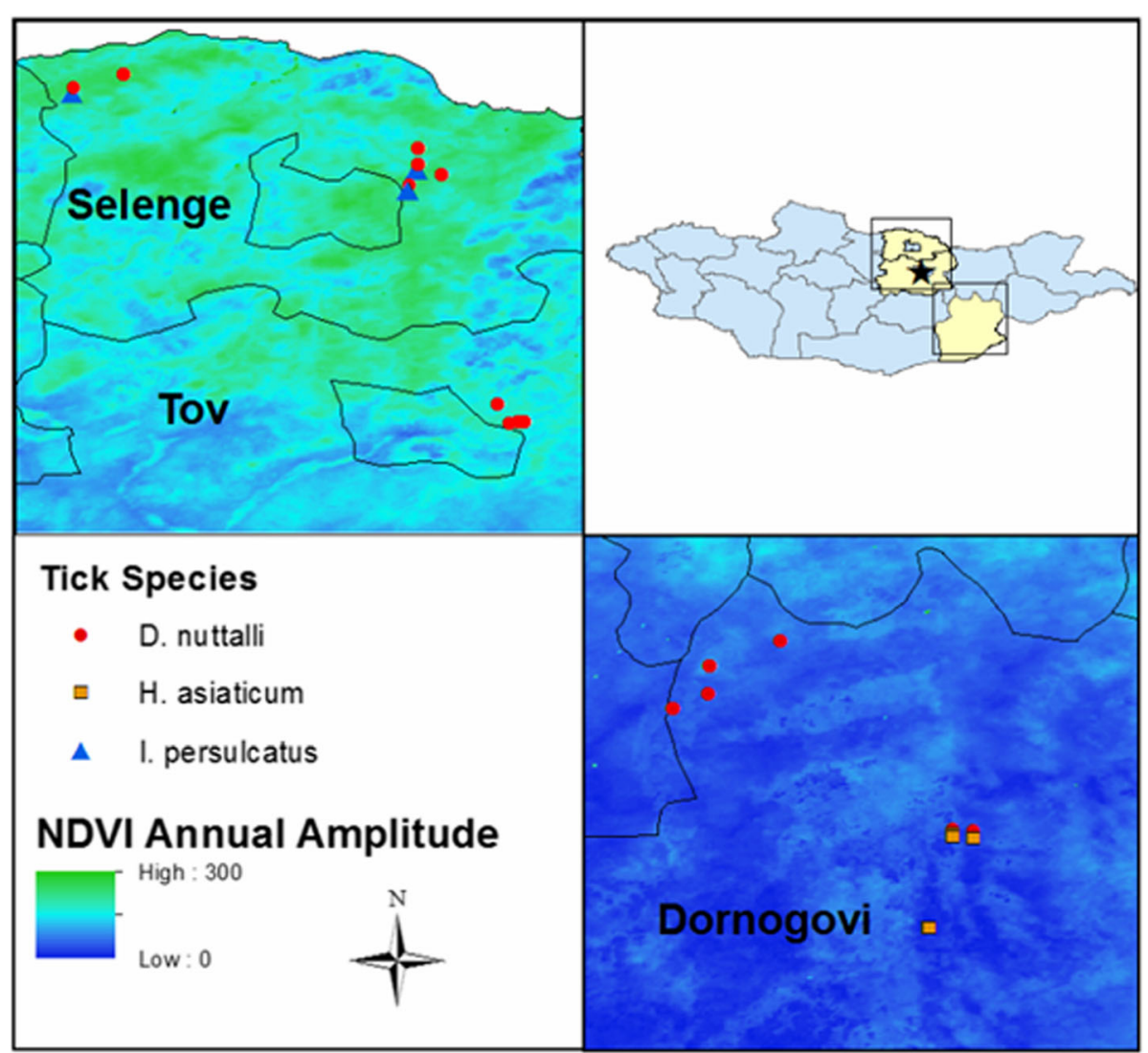

Fig. 1 Tick species distribution overlaid on vegetation coverage

MIR ranging from 11.2 to $16.6 \%$. The MLE of "Candidatus R. tarasevichiae" in I. persulcatus ticks was $46.6 \%$ (95\% CI: $35.2-63.6 \%)$ compared to a MIR of $19.5 \%$ (95\% CI: 14.7-24.4\%). All 88 pools of $H$. asiaticum ticks tested negative for Rickettsia DNA. All "Candidatus R. tarasevichiae"-infected ticks were collected in Selenge aimag, which is a known habitat for I. persulcatus in Mongolia $[7,19,20]$.

Based on the phylogenetic analysis of $341 \mathrm{bp}$ gltA gene sequence data, we documented two sequences of $R$. raoultii and one "Candidatus $\mathrm{R}$. tarasevichiae" sequence. One of the $R$. raoultii sequences (KU361213) shared

Table 2 Summary of molecular study results from ticks collected in Mongolia April 24th and May 16th 2015

\begin{tabular}{|c|c|c|c|c|}
\hline \multirow[t]{2}{*}{ Tick species } & \multirow[t]{2}{*}{ Soum (aimag) } & \multicolumn{3}{|c|}{ Pathogens detected (\%) } \\
\hline & & R. raoultii & "Candidatus R. tarasevichiae" & TBEV \\
\hline \multirow[t]{6}{*}{ D. nuttalli } & Terelj (Tov) & $60 / 71(84.5)$ & 0 & $0 / 46$ \\
\hline & Dalanjargalan (Dornogovi) & $29 / 51(56.8)$ & 0 & nd \\
\hline & Eroo (Selenge) & $21 / 28(75)$ & 0 & $0 / 28$ \\
\hline & Sainshand (Dornogovi) & $1 / 2(50)$ & 0 & nd \\
\hline & Tushig (Selenge) & $4 / 6(66.6)$ & 0 & $0 / 6$ \\
\hline & Totals & 115/158 (72.8) & 0 & $0 / 80$ \\
\hline H. asiaticum & Sainshand (Dornogovi) & 0 & 0 & nd \\
\hline \multirow[t]{3}{*}{ 1. persulcatus } & Eroo (Selenge) & 0 & $48 / 52(92.3)$ & $1 / 52(1.9)$ \\
\hline & Tushig (Selenge) & 0 & $1 / 1(100)$ & $0 / 1$ \\
\hline & Totals & 0 & $49 / 53(92.5)$ & 1/53 (1.9) \\
\hline Totals & & $115 / 299(38.5)$ & 49/299 (16.4) & $1 / 133(0.1)$ \\
\hline
\end{tabular}


Table 3 Using minimum infection rates and maximum likelihood estimations to calculate tick infection rates by species and aimag

\begin{tabular}{lllll}
\hline Aimag & Species & Pathogen & Minimum infection rate $(95 \%$ Cl) & Maximum likelihood infection rate (95\% Cl) \\
\hline Selenge & I. persulcatus & "Candidatus R. tarasevichiae" & $19.5(14.7-24.4)$ & $46.6(35.2-63.6)$ \\
Selenge & D. nuttalli & R. raoultii & $14.8(9.4-20.2)$ & $22.6(15.5-32.2)$ \\
Tov & D. nuttalli & R. raoultii & $16.6(12.9-20.6)$ & $30.4(23.8-38.6)$ \\
Dornogovi & D. nuttalli & R. raoultii & $11.2(7.4-15.0)$ & $15.1(10.6-20.9)$ \\
\hline
\end{tabular}

$100 \%$ identify with $R$. raoultii (JX945524) which was detected in two patients in China [21], with the other sequence (KU361214) differing from the Chinese sequence by one base. The "Candidatus R. tarasevichiae" of our study (KU361212) shared 100\% identity with the sequence JX996054 recovered from human cases of "Candidatus R. tarasevichiae" in China [15]. Phylogenetic analysis based on $322 \mathrm{bp}$ ompA gene sequence data determined one of two $R$. raoultii sequences (KU361215) and the only "Candidatus R. tarasevichiae" sequence (KU361217) recovered in our study shared 100\% identity with sequences JX945525 and JX996053 from human cases in China $[15,22]$. The other $R$. raoultii sequence showed $99.4 \%$ similarity to that of the human patient in China with 2 bp difference. Phylogenetic trees depicting variation by species and gene fragment can be found in Figs. 2 and 3.

Previous entomological surveys focusing on ticks in Mongolia have reported a countrywide distribution of $D$. nuttalli, while $H$. asiaticum and $I$. persulcatus have clearly defined ecological niches on opposite sides of
Mongolia [23], thus influencing the geographical distribution of species-specific tick-borne diseases. Living in close proximity to coniferous regions in the northern aimags of Mongolia likely remains a significant risk factor for exposure to diseases transmitted by I. persulcatus hard ticks [7, 11, 12, 19, 20, 24-26]. Rickettsia raoultii detection among $D$. nuttalli ticks were observed in all five sampling districts, suggesting a wider distribution of disease, even extending into China as indicated by other reports [21, 27]. These findings expand upon previous findings which described $R$. raoultii as the predominant SFG Rickettsia infection found in $179 \mathrm{D}$. nuttalli ticks collected in four northern Mongolian provinces [7].

\section{Conclusion}

The presence of "Candidatus R. tarasevichiae" in ticks should be of concern, given that the ompA and gltA sequences from this study shared $100 \%$ identity with severely ill cases of "Candidatus R. tarasevichiae" infection that were recently described $[15,22]$. To date there has only been one other study that has reported evidence

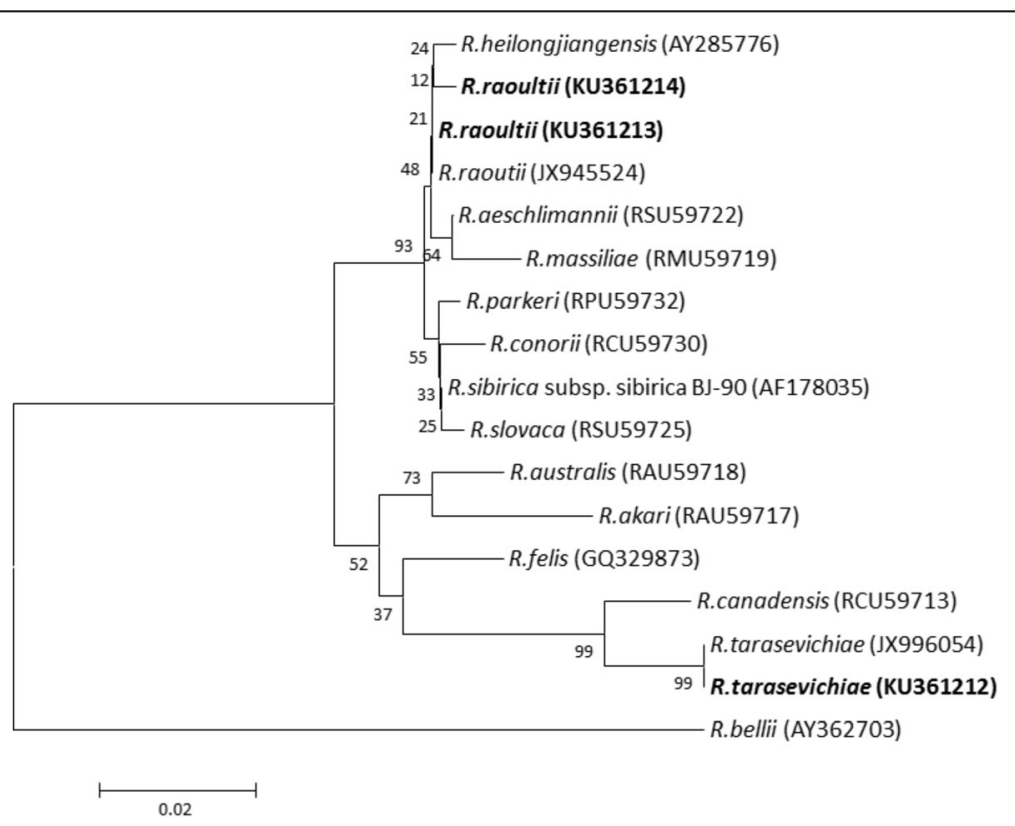

Fig. 2 Phylogenetic analysis was conducted based on 341-bp gltA gene sequence data with the neighbour-joining method under a Kimura 2-parameter model using MEGA software, version 5.0. Bootstrap analysis of 10,000 replicates was oriented by using R. bellii as the outgroup. Numbers on the branches indicate percentage of replicates that reproduced the topology for each clade. Scale-bar indicates estimated evolutionary distances. The GenBank accession number is listed at the end of each strain. The sequences in bold are the ones identified in this study 


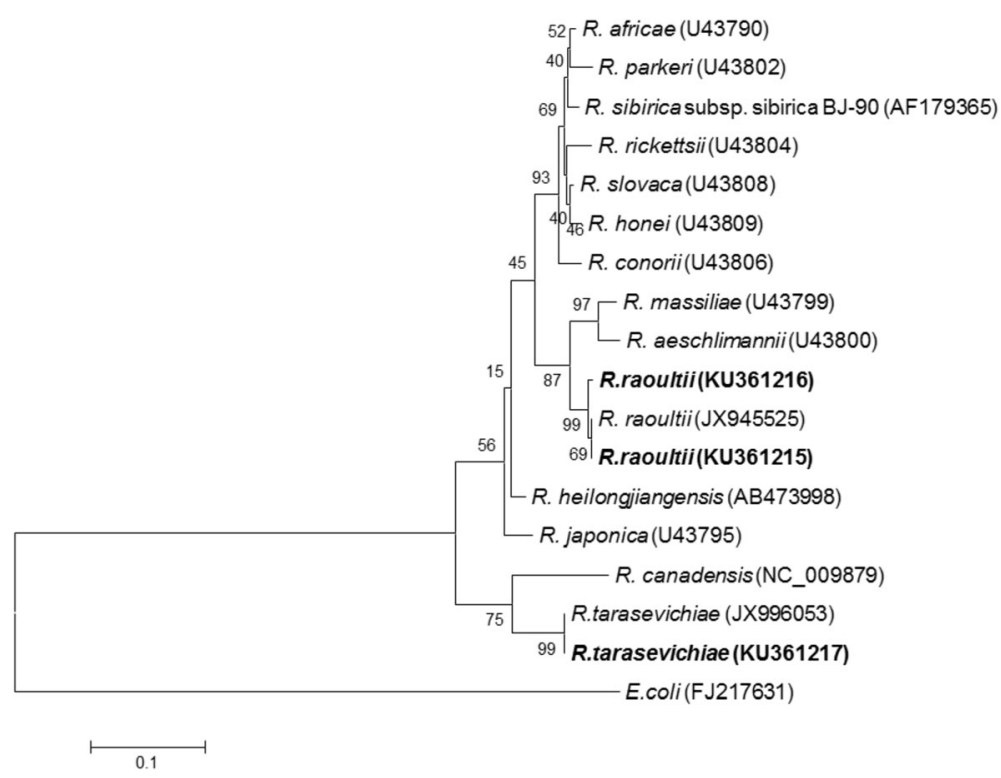

Fig. 3 Phylogenetic analysis was conducted based on 322-bp outer membrane protein A (ompA) gene sequence data with the neighbour-joining method under a Kimura 2-parameter model using MEGA software, version 5.0. Bootstrap analysis of 10,000 replicates was oriented by using E. coli as the outgroup. Numbers on the branches indicate percentage of replicates that reproduced the topology for each clade. Scale-bar indicates estimated evolutionary distances. The GenBank accession number is listed at the end of each strain. The sequences in bold are the ones identified in this study

of "Candidatus R. tarasevichiae" in Mongolia [8]. While little is known about the full clinical spectrum of this disease, some patients in China infected with "Candidatus $\mathrm{R}$. tarasevichiae" presented with meningitis-like symptoms that are atypical to SFG rickettsiosis, resulting in initial misdiagnosis. This may prove problematic in Mongolia, where TBEV infections commonly presents with fever and meningitis-like symptoms, and is considered a high risk disease in Selenge. However, our analysis of ticks in this region indicated only one positive I. persulcatus pool for TBEV (1.9\%), which matches similar reports of TBEV from other studies in this region $[11,19]$. This is in contrast to the $92.5 \%$ positive pool detection prevalence or 46.6\% tick infection rate based on MLE, of "Candidatus R. tarasevichiae" from the same tick pools.

Future studies are needed to determine if "Candidatus R. tarasevichiae" or $R$. raoultii are causing clinical disease in Mongolia. Until then, both Mongolian nationals and foreign travellers who visit the countryside during late spring and summer, should take additional precautions to prevent exposure to ticks and be aware of the risks they pose. Mongolian physicians treating suspected cases of TBEV should include "Candidatus R. tarasevichiae" infection in their differential diagnosis and consider prescribing antimicrobial therapy.

\section{Acknowledgments}

This study was made possible due to the assistance and support provided by the National Center for Zoonotic Diseases (NCZD), the Beijing Institute of Microbiology and Epidemiology (BIME), and the Mongolian Academy of
Medical Sciences (MAMS). We thank Sophia Papageorgiou, for her early contributions to this study and Dr. Enkhamgalan for her ongoing administrative support.

\section{Funding}

Funding for this work was provided by the National Key Technology Support Program of China (JF Jiang PI) and by the National Institutes of Health, Fogarty International Center grant, D43TW009373, "One Health Innovation Fellowships for Zoonotic Disease Research in Mongolia" (GC Gray PI).

\section{Availability of data and materials}

Sequences were submitted to the GenBank database under accession numbers KU361212-KU361217.

\section{Authors' contributions}

Study design: BB, SL, PN and GCG; sample collection: BB and SL; Lab analysis: $\mathrm{RJ}, \mathrm{BB}, \mathrm{MVF}, \mathrm{BB}, \mathrm{YW}$ and JJ; manuscript preparation: MV, RJ, BA and GCG. All authors read and approved the final manuscript.

\section{Competing interests}

The authors declare that they have no competing interests.

\section{Consent for publication}

Not applicable.

\section{Ethics approval and consent to participate}

Not applicable.

\section{Author details}

${ }^{1}$ Institute of Veterinary Medicine, Ulaanbaatar, Mongolia. ${ }^{2}$ State Key Laboratory of Pathogen and Biosecurity, Beijing Institute of Microbiology and Epidemiology, Beijing, China. ${ }^{3}$ Division of Infectious Disease, Duke Global Health Institute, Duke University, Durham, NC, USA. ${ }^{4}$ Department of Global and Community Health, George Mason University, Fairfax, VA, USA. ${ }^{5}$ Mongolian Academy of Medical Sciences, Ulaanbaatar, Mongolia. ${ }^{6}$ National Center for Zoonotic Diseases, Ulaanbaatar, Mongolia. 
Received: 25 October 2016 Accepted: 12 January 2017

\section{Published online: 02 February 2017}

\section{References}

1. Dantas-Torres F, Chomel BB, Otranto D. Ticks and tick-borne diseases: a one health perspective. Trends Parasitol. 2012;28(10):437-46.

2. Pfäffle M, Littwin N, Muders SV, Petney TN. The ecology of tick-borne diseases. Int J Parasitol. 2013;43(12-13):1059-77.

3. Parola P, Paddock CD, Socolovschi C, Labruna MB, Mediannikov O, Kernif T, et al. Update on tick-borne rickettsioses around the world: a geographic approach. Clin Microbiol Rev. 2013;26(4):657-702.

4. Fang LQ, Liu K, Li XL, Liang S, Yang Y, Yao HW, et al. Emerging tick-borne infections in mainland China: an increasing public health threat. Lancet Infect Dis. 2015;15(12):1467-79.

5. Eremeeva ME, Dasch GA. Challenges posed by tick-borne rickettsiae: ecoepidemiology and public health implications. Front Public Health. 2015;3:55.

6. Lewin MR, Bouyer DH, Walker DH, Musher DM. Rickettsia sibirica infection in members of scientific expeditions to northern Asia. Lancet. 2003;362(9391): $1201-2$.

7. Speck S, Derschum H, Damdindorj T, Dashdavaa O, Jiang J, Kaysser $P$, et al. Rickettsia raoultii, the predominant Rickettsia found in Mongolian Dermacentor nuttalli. Ticks Tick Borne Dis. 2012:3(4):227-31.

8. Anu D, Gill BC, Lee SE, Hong SH, Sh CH, Lee WJ, et al. Molecular detection of Rickettsia species in some ticks in Mongolia. Mongolian J Infect Dis. 2015; 4(63):3-6.

9. Hayasaka D, Ivanov L, Leonova GN, Goto A, Yoshii K, Mizutani T, et al. Distribution and characterization of tick-borne encephalitis viruses from Siberia and far-eastern Asia. J Gen Virol. 2001;82(6):1319-28.

10. Leonova GN, Maystrovskaya OS, Kondratov IG, Takashima I, Belikov SI. The nature of replication of tick-borne encephalitis virus strains isolated from residents of the Russian far east with inapparent and clinical forms of infection. Virus Res. 2014;189:34-42.

11. Muto M, Bazartseren B, Tsevel B, Dashzevge E, Yoshii K, Kariwa H. Isolation and characterization of tick-borne encephalitis virus from Ixodes persulcatus in Mongolia in 2012. Ticks Tick Borne Dis. 2015:6(5):623-9.

12. Walder G, Lkhamsuren E, Shagdar A, Bataa J, Batmunkh T, Orth D, et al. Serological evidence for tick-borne encephalitis, borreliosis, and human granulocytic anaplasmosis in Mongolia. Int J Med Microbiol. 2006;296:69-75.

13. Hay SI, Tatem AJ, Graham AJ, Goetz SJ, Rogers DJ. Global environmental data for mapping infectious disease distribution. Adv Parasitol. 2006;62:37-77.

14. Black WC, Piesman J. Phylogeny of hard- and soft-tick taxa (Acari: Ixodida) based on mitochondrial 165 rDNA sequences. Proc Natl Acad Sci USA. 1994; 91(21):10034-8.

15. Jia N, Zheng YC, Jiang JF, Ma L, Cao WC. Human infection with Candidatus Rickettsia tarasevichiae. N Engl J Med. 2013;369(12):1178-80.

16. Mediannikov OY, Sidelnikov Y, Ivanov L, Mokretsova E, Fournier PE, Tarasevich I, et al. Acute tick-borne rickettsiosis caused by Rickettsia heilongjiangensis in the Russian far east. Emerg Infect Dis. 2004;10(5):810-7.

17. Pacheco RC, Moraes-Filho J, Marcili A, Richtzenhain $\sqcup$, Szabó MPJ, Catroxo $\mathrm{MHB}$, et al. Rickettsia monteiroi sp. nov., infecting the tick Amblyomma incisum in Brazil. Appl Environ Microbiol. 2011;77(15):5207-11.

18. Regnery RL, Spruill CL, Plikaytis BD. Genotypic identification of rickettsiae and estimation of intraspecies sequence divergence for portions of two rickettsial genes. J Bacteriol. 1991;173(5):1576-89.

19. Frey S, Mossbrugger I, Altantuul D, Battsetseg J, Davaadorj R, Tserennorov D, et al. Isolation, preliminary characterization, and full-genome analyses of tick-borne encephalitis virus from Mongolia. Virus Genes. 2012;45(3):413-25.

20. Javkhlan G, Enkhtaivan B, Baigal B, Myagmarsuren P, Battur B, Battsetseg B. Natural Anaplasma phagocytophilum infection in ticks from a forest area of Selenge province, Mongolia. West Pac Surveill Response J. 2014;5(1):21-4.

21. Jia N, Zheng $Y C, M a L, H u o ~ Q B, N i X B$, Jiang BG, et al. Human infections with Rickettsia raoultii, China. Emerg Infect Dis. 2014;20(5):866-8.

22. Liu W, Li H, Lu QB, Cui N, Yang ZD, Hu JG, et al. Candidatus Rickettsia tarasevichiae infection in eastern Central China: a case series. Ann Intern Med. 2016;164(10):641-8

23. Dash M, Biambaa B, Neronov V. The ixodid tick fauna of the Mongolian People's Republic. I. The species distribution. Med Parazitol (Mosk). 1988:(3):37-42.

24. Masuzawa T, Masuda S, Fukui T, Okamoto Y, Bataa J, Oikawa Y, et al. PCR detection of Anaplasma phagocytophilum and Borrelia burgdorferi in IXodes persulcatus ticks in Mongolia. Jpn J Infect Dis. 2014;67(1):47-9.
25. Scholz HC, Margos G, Derschum H, Speck S, Tserennorov D, Erdenebat N, et al. High prevalence of genetically diverse Borrelia bavariensis-like strains in Ixodes persulcatus from Selenge Aimag, Mongolia. Ticks Tick Borne Dis. 2013; 4(1-2):89-92.

26. Tuvshintulga B, Sivakumar T, Battsetseg B, Narantsatsaral SO, Enkhtaivan B, Battur B, et al. The PCR detection and phylogenetic characterization of Babesia microti in questing ticks in Mongolia. Parasitol Int. 2015;64(6):527-32.

27. Wen J, Jiao D, Wang JH, Yao DH, Liu ZX, Zhao G, et al. Rickettsia raoultii, the predominant Rickettsia found in Dermacentor silvarum ticks in China-Russia border areas. Exp Appl Acarol. 2014;63(4):579-85.

\section{Submit your next manuscript to BioMed Central and we will help you at every step:}

- We accept pre-submission inquiries

- Our selector tool helps you to find the most relevant journal

- We provide round the clock customer support

- Convenient online submission

- Thorough peer review

- Inclusion in PubMed and all major indexing services

- Maximum visibility for your research

Submit your manuscript at www biomedcentral.com/submit
C Biomed Central 